\title{
Avaliação da resposta do gel polimérico MAGIC- $f$ modificado utilizando uma fonte clínica de braquiterapia e simulação Monte Carlo com o pacote PENELOPE
}

Evaluation of the response of modified MAGIC-f polymeric gel using a clinical brachytherapy source and Monte Carlo simulation with package

PENELOPE

\author{
Ana Luíza Quevedoํㄹ Leandro F. Borges², Patrícia Nicolucci ${ }^{1}$ \\ ${ }^{1}$ Universidade de São Paulo / Departamento de Física, Faculdade de Filosofia Ciências e Letras de \\ Ribeirão Preto, Ribeirão Preto, Brasil \\ ${ }^{2}$ Setor de Radioterapia, Hospital das Clínicas da Faculdade de Medicina de Ribeirão Preto da \\ Universidade de São Paulo, Ribeirão Preto, Brasil
}

\begin{abstract}
Resumo
Neste trabalho foi realizada a comparação entre distribuições de doses relativas experimental e simulada de uma fonte clínica de braquiterapia. Um objeto simulador de $5 \times 5 \times 7 \mathrm{~cm}^{3}$ com gel polimérico MAGIC-f, com formulação modificada, foi irradiado com uma fonte clínica de ${ }^{192}$ Ir, Varian, modelo GammaMed Plus. Esse objeto simulador foi irradiado com uma dose de 7,5 Gy, comumente utilizada em tratamentos ginecológicos. A leitura da distribuição de dose foi realizada $24 \mathrm{~h}$ após a irradiação, utilizando-se Imagem por Ressonância Magnética, ponderada em T2, com tempo de repetição de $6000 \mathrm{~ms}, 15 \mathrm{~ms}$ de tempo ao eco, 16 ecos e tamanho de pixel de 0,40 mm. Essa mesma fonte foi modelada no pacote PENELOPE e utilizada para a obtenção das distribuições de doses da mesma. As energias de corte para elétrons, fótons e pósitrons foram de $100 \mathrm{keV}$, os parâmetros de condensão de partículas primárias permaneceram iguais à 0,3 . Foram obtidas distribuições de dose em dois planos perpendiculares à fonte: um plano passando pelo centro da fonte e outro à $0,5 \mathrm{~cm}$ do centro da fonte na direção do topo do encapsulamento. Comparando-se os resultados experimentais utilizando o gel polimérico aos resultados computacionais, as maiores diferenças entre as distribuições de doses foram de $12,5 \%$ em um ponto a 0,62 cm da fonte, para o plano passando pelo centro da fonte, e de $6,4 \%$ a $0,5 \mathrm{~cm}$, para o plano superior. Considerando-se o alto gradiente de dose de fontes clínicas de Braquiterapia, os resultados obtidos nesse trabalho indicam que 0 gel MAGIC-f com sua formulação modificada, apresenta-se como promissor para dosimetria em braquiterapia.
\end{abstract}

Palavras-chave: Braquiterapia; gel polimérico; Simulação Monte Carlo; PENELOPE.

\begin{abstract}
In this work a comparison of experimental and simulated relative doses of a clinical brachytherapy source was performed. A $5 \times 5 \times 7 \mathrm{~cm}^{3}$ phantom with a modified MAGIC-f gel was irradiated using a clinical ${ }^{192} / \mathrm{r}$ source, Varian, model GammaMed Plus. The phantom was irradiated with 7,5 Gy, commonly used in gynecological treatments. The dose distributions readings were performed $24 \mathrm{~h}$ after irradiation using Magnetic Resonance Imaging, weighted in T2, with repetition time of $6000 \mathrm{~ms}$, echo-time of $15 \mathrm{~ms}, 16$ echos and $0.40 \mathrm{~mm}$ pixel size. The same brachytherapy source was modeled in the Monte Carlo package PENELOPE to obtain dose distributions. The energy cutoff for electrons, photons and positrons was $100 \mathrm{keV}$, the primary particles condensation parameters remained the same at 0.3 . The dose distributions were obtained in two planes perpendicular to the source: one passing through the source's center and the other at $0.5 \mathrm{~cm}$ away from the source's center in direction of the top of encapsulation. A comparison of the experimental results using the polymeric gel to the computational results showed maximum differences of $12.5 \%$, at $0.62 \mathrm{~cm}$ from the source for the central plane, and $6.4 \%$ at $0.5 \mathrm{~cm}$ from the source for the superior plane. Considering the high dose gradient of these clinical brachytherapy sources, the results obtained in these work show that MAGIC-f gel with modified formulation, is promising for dosimetry in brachytherapy.
\end{abstract}

Keywords: Brachytherapy; polymer gel; Monte Carlo Simulation; PENELOPE.

\section{Introdução}

Um sistema de planejamento, tipicamente utilizado em tratamentos de radioterapia, é alimentado por informações dosimétricas a respeito da fonte, incluindo dados a respeito da geometria, atenuação e espalhamento do feixe ${ }^{1,2}$. Tais informações dosimétricas podem ser obtidas de forma analítica, experimental e computacional, dependendo da função dosimétrica analisada. Um dos métodos que pode ser utilizado na obtenção 
de características dosimétricas de fontes de braquiterapia é a simulação Monte Carlo com o código PENELOPE, que simula o transporte de elétrons, pósitrons e fótons em materiais e geometrias arbitrários ${ }^{3}$. Esse código utiliza na sua implementação algoritmos denominados classe II, ou algoritmo misto. Esse tipo de algoritmo realiza simulações detalhadas no caso de as partículas amostradas serem fótons. Caso as partículas amostradas sejam elétrons ou pósitrons, a simulação poderá ser realizada de forma detalhada, caso existam eventos de interação fortes, ou de forma condensada, caso ocorram espalhamentos em baixo ângulo.

Devido ao alto gradiente de dose em braquiterapia, determinações de doses experimentais são difíceis de serem realizadas. Alguns dosímetros podem ser utilizados na determinação experimental de dose em radioterapia, porém apresentam algumas limitações em braquiterapia. Dentre esses dosímetros, podemos citar a câmara de ionização, os dosímetros termoluminescentes (TLDs) e filmes radiocrômicos ${ }^{4-7}$. Através do uso dos dois primeiros, é possível obter medidas pontuais, enquanto que 0 segundo permite obter distribuições bidimensionais.

A dosimetria em braquiterapia requer o uso de dosímetros que possuam alta sensibilidade, alta resolução espacial e pouca dependência energética $^{8,9}$. Nesse sentido, a dosimetria gel polimérica tem ganhado destaque nessas aplicações, uma vez que é possível obter distribuições de dose tridimensionais, com alta resolução espacial e alta sensibilidade ${ }^{10}$. $O$ gel polimérico utilizado nesse estudo foi o MAGIC- $f$ (do inglês, Methacrylic Ascorbic Acid and Gelatine Initiated by Cooper Solution with Formaldehyde), que permite a obtenção de dados com alta resolução espacial, sendo capaz de proporcionar uma distribuição de dose tridimensionall11,12. No momento que o gel é irradiado, dá-se início ao processo de polimerização, onde há uma mudança na matriz do gel. Essa mudança, proporcional ao valor da dose, poderá ser quantificada utilizando Imagens de Ressonância Magnética, ultrassom e tomografias computadorizadas ${ }^{13,14}$ Nesse trabalho, a técnica de leitura do gel utilizada foi através da Imagem de Ressonância Magnética.

Neste trabalho, foram obtidas as distribuições de dose de uma fonte clínica de ${ }^{192}$ Ir utilizada em braquiterapia, através do uso do gel polimérico MAGIC-f com sua formulação modificada. Os perfis de dose, em planos passando pelo centro da fonte e à $0,5 \mathrm{~cm}$ na direção do topo do encapsulamento, foram comparados com os valores obtidos utilizando simulação Monte Carlo com o pacote PENELOPE.

\section{Materiais e Métodos}

Neste trabalho, a simulação Monte Carlo com o pacote PENELOPE foi utilizada na obtenção das distribuições de dose de uma fonte de ${ }^{192}$ Ir de alta taxa de dose. Os resultados obtidos computacionalmente foram comparados com os resultados experimentais, utilizando 0 gel polimérico MAGIC-f com sua formulação modificada.

\subsection{Simulação Monte Carlo com o pacote PENELOPE}

O material radioativo dessa fonte possui aproximadamente 40 linhas de energia com valor médio igual à $397 \mathrm{keV}^{15,16}$. O espectro de radiação primário, utilizado nas simulações, foi composto pelas 17 linhas de emissão de fótons mais prováveis, presentes no intervalo de $65 \mathrm{keV}$ à 1378 keV $^{17}$.

A geometria real de uma fonte clínica de ${ }^{192} \mathrm{Ir}$, Varian, modelo GammaMed Plus, foi construída de acordo com as dimensões citadas por Baltas e colaboradores $^{18}$, composta por parte ativa, encapsulamento e cabo. A parte ativa da fonte modelada computacionalmente, possuindo raio de $0,03 \mathrm{~cm}$ e comprimento de $0,35 \mathrm{~cm}$. O encapsulamento, de aço inoxidável AISI 316L, possui raio de $0,055 \mathrm{~cm}$ e comprimento de $0,5 \mathrm{~cm}$. O cabo, também de aço inoxidável AISI 316L, possui raio de $0,055 \mathrm{~cm}$ e comprimento de $150 \mathrm{~cm}$. Nos tratamentos de braquiterapia ginecológica são utilizados aplicadores com o objetivo de guiar a fonte até o volume tumoral que será tratado. O aplicador simulado é de aço inoxidável AISI 316L e possui comprimento de $20 \mathrm{~cm}$ e diâmetro de 0,165 $\mathrm{cm}$, idêntico ao utilizado na etapa experimental.

Os dados das seções de choque dos materiais utilizados nas simulações, foram obtidos junto ao pacote de dados do código PENELOPE. Os dados das seções de choque para o aço inoxidável AISI 316L, material que é feito o aplicador e que compõe o encapsulamento e o cabo da fonte, não existem no banco de dados do código PENELOPE. Portanto, foi construído um arquivo desse material a partir de informações químicas e estequiométricas fornecidas no artigo de Baltas e colaboradores $^{18}$. Nas simulações realizadas para a obtenção das distribuições de dose, a fonte foi posicionada no centro de um objeto simulador cúbico, homogêneo, de $30 \mathrm{~cm}$ de lado. Foi escolhida uma distância total de $6 \mathrm{~cm}$ nas três direções do eixo cartesiano, que foi dividido em 101 voxels para a obtenção das informações dosimétricas, com um voxel na posição central da geometria. Desta forma, a dimensão dos voxels foi de $0,0594 \mathrm{~cm}$.

O número de partículas amostradas permaneceu constante em $10^{9}$ e a energia de corte para fótons e elétrons foi de $100 \mathrm{keV}$. Todas as distribuições de dose foram obtidas em planos transversais passando pelo centro da fonte de ${ }^{192} \mathrm{Ir}$ e os valores das doses foram normalizadas no primeiro voxel fora da fonte. Foram obtidos os valores de dose relativa no plano central da fonte $(z=0 \mathrm{~cm})$ e à $0,5 \mathrm{~cm}$ na direção do topo do encapsulamento $(z=+0,5 \mathrm{~cm})$ e a normalização 
das doses em cada plano ocorreu em relação ao primeiro voxel fora da fonte.

\subsection{Gel Polimérico MAGIC-f modificado}

O gel polimérico utilizado nesse estudo, MAGIC$f$, é composto basicamente por gelatina, água milli$Q$, solução de sultafo de cobre, ácido ascórbico, ácido metacrílico e formaldeído. Esse gel possui diversas vantagens com relação aos outros géis poliméricos já estudados, dentre elas: a supressão do oxigênio pelo ácido ascórbico, o que resulta em um gel com vantagens práticas para possíveis aplicações na clínica e menor toxicidade; alta resolução espacial. A manufatura desse gel foi a mesma de Pavoni e colaboradores ${ }^{19}$, porém, as concentrações dos reagentes foram modificadas para: $11 \mathrm{~g}$ de Gelatina, $2 \mathrm{ml}$ da Solução de Sulfato de Cobre, 50,2 mg de Ácido Ascórbico, 5,9 ml de Ácido Metacrílico e $4 \mathrm{ml}$ de Formaldeído.

Os objetos simuladores utilizados nas irradiações, foram tubos Vacutainerß de $5 \mathrm{ml}$, para a construção da curva de calibração, e recipiente de acrílico de dimensões $5 \times 5 \times 7(\mathrm{~cm})^{3}$, para a obtenção da distribuição de dose. Após a inserção do gel no objeto simulador, o mesmo foi recoberto com uma camada de filme plástico de PVC, para evitar o contato do oxigênio com o gel e em seguida todos os objetos simuladores foram estocados em uma geladeira à $10^{\circ} \mathrm{C}$. A construção da curva de calibração foi realizada com doses em um intervalo de 0 à 30 Gy, com 3 tubos irradiados simultaneamente para cada dose. O objeto simulador de estudo foi irradiado com uma dose de 7,5 Gy.

\subsection{Leitura das Amostras por Imagem de Ressonância Magnética}

As mudanças causadas na matriz do material, a partir do momento da irradiação, podem ser quantificadas através de diversas técnicas. Neste trabalho, a leitura do gel foi realizada através da imagem por ressonância magnética. A leitura de todos os objetos simuladores foram realizadas em um tomógrafo Philips de 3.0 T, 24 horas após a irradiação e em uma bobina de cabeça de 32 canais. Em todas as aquisições, as imagens foram obtidas utilizando relaxometria em T2, com tempo de repetição de $6000 \mathrm{~ms}$ e tempo ao eco de $15 \mathrm{~ms}$.

Para os objetos simuladores menores, foram realizados cortes passando pelo centro da fonte, com espessura de $2 \mathrm{~mm}$. Para a obtenção da distribuição de dose do objeto simulador maior, foi realizado um corte passando pelo centro da fonte $(z=0 \mathrm{~cm})$ e outro à $0,5 \mathrm{~cm}$ na direção do topo do encapsulamento da mesma $(z=+0,5 \mathrm{~cm})$.

\section{Resultados}

$\mathrm{Na}$ figura 1 é apresentada a imagem de ressonância magnética do plano transversal dos tubos Vacutainer®, referentes à irradiação para obtenção da curva de calibração.

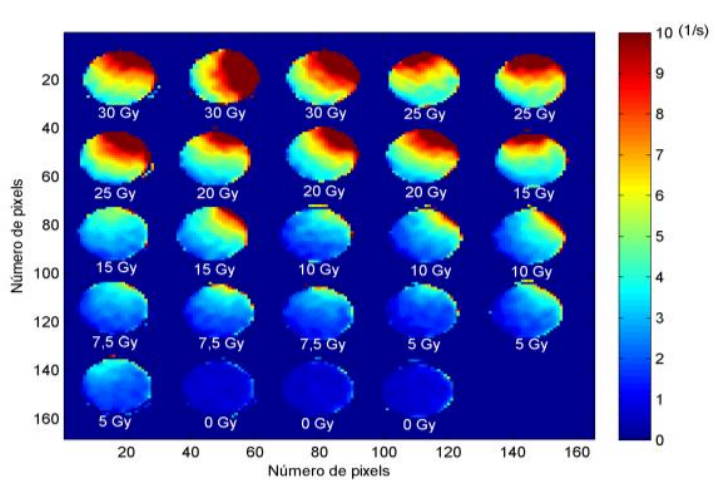

Figura 1: Imagem de ressonância magnética nuclear dos tubos Vacutainer®, referentes à curva de calibração.

Na figura 2 são apresentados os valores médios de R2 em função das doses, referente à irradiação dos tubos Vacutainer®.

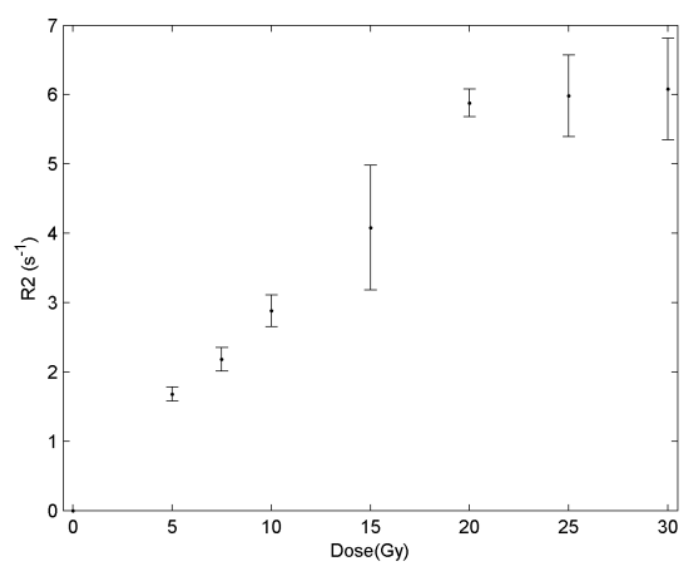

Figura 2: Valores médios de R2, em função das doses, referente aos tubos Vacutainer®.

Como é possível observar a partir da figura 2, entre as doses 20 Gy e 30 Gy, há uma saturação na resposta do dosímetro. Os mapas de R2, referentes ao objeto simulador maior, com cortes passando pelo centro da fonte $(z=0 \mathrm{~cm})$ e à $0,5 \mathrm{~cm}$ na direção do topo do encapsulamento da fonte $(z=+0,5 \mathrm{~cm})$, são apresentadas na figura 3 (a) e (b), respectivamente.

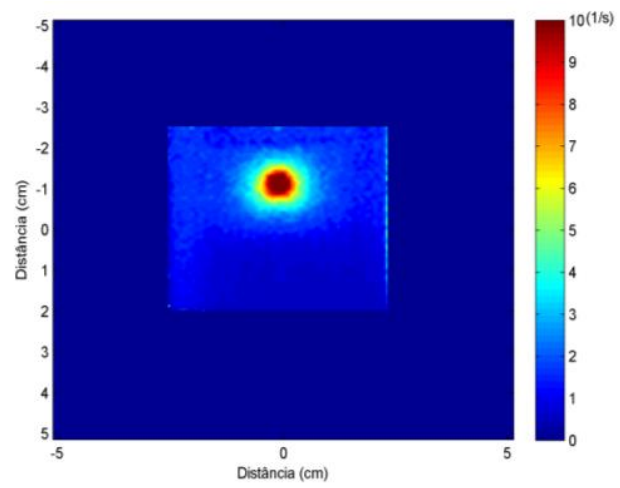

(a) 


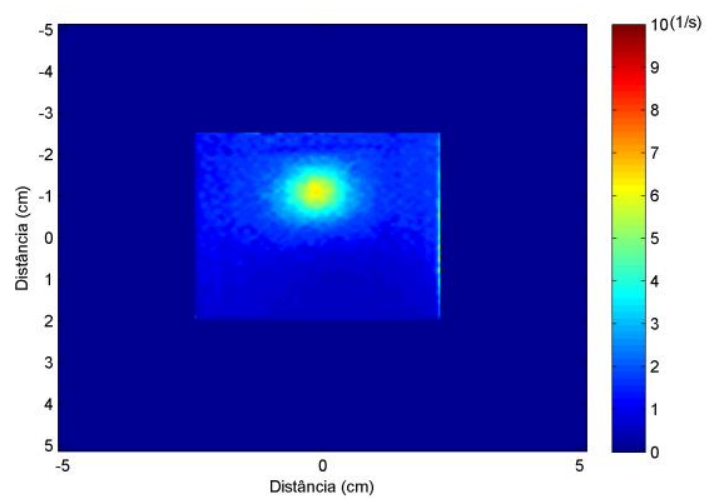

(b)

Figura 3: Mapas dos valores de R2 referentes aos cortes passando (a) pelo centro da fonte $(z=0 \mathrm{~cm})$ e $(\mathrm{b})$ à $0,5 \mathrm{~cm}$ do centro da fonte $(z=+0,5 \mathrm{~cm})$.

Para a comparação entre os dados obtidos computacionalmente e experimentalmente, foram selecionados planos passando pelo centro das imagens das figuras 3 e normalizados pelo primeiro voxel fora da fonte. A comparação entre os dados computacionais e experimentais, são apresentados na figura 4 (a) e (b), referentes ao plano central e à $0,5 \mathrm{~cm}$ do centro da fonte, respectivamente.

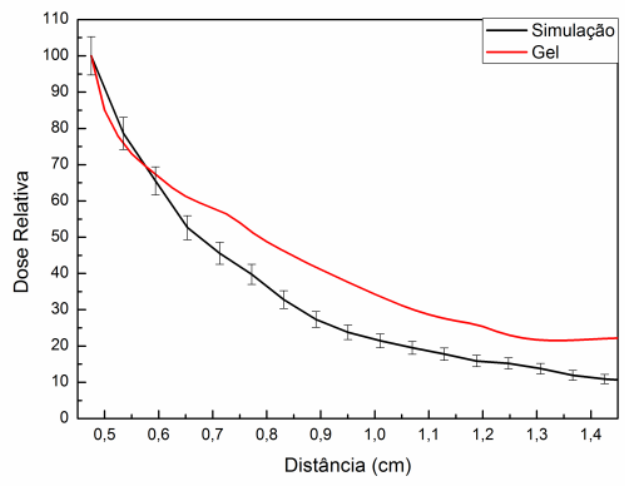

(a)

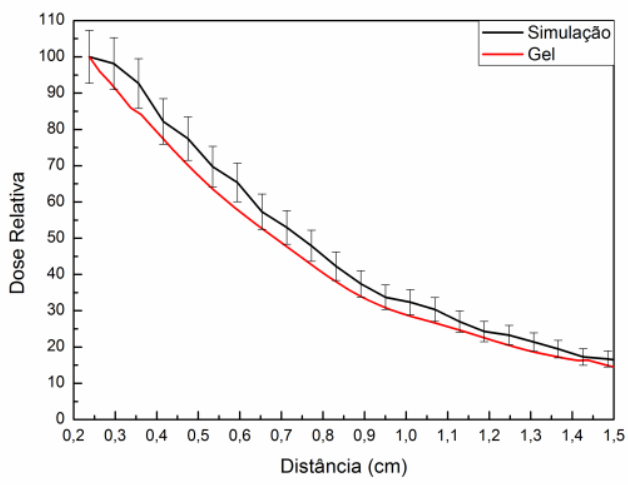

(b)

Figura 4: Dose relativa em função da distância referentes (a) ao plano central e (b) à $0,5 \mathrm{~cm}$ do centro da fonte.
Para a comparação referente ao plano central da fonte, a maior diferença encontrada entre os dados computacionais e os experimentais, foi de $12,5 \%$ a $0,62 \mathrm{~cm}$ da fonte na direção radial. Com relação à comparação entre os valores referentes ao plano à $0,5 \mathrm{~cm}$ do centro da fonte, a maior diferença encontrada foi de $6,4 \%$ a $0,5 \mathrm{~cm}$ da fonte na direção radial.

\section{Discussão}

$\mathrm{Na}$ construção da curva de calibração do gel polimérico MAGIC- $f$, pode-se observar uma, saturação na resposta do gel no intervalo de 20 à 30 Gy. Porém, os valores da resposta do gel na região de 0 a 15 Gy, podem ser ajustados de forma linear, com índice de correção entre os pontos de 0,997 .

Com relação às comparações da resposta do gel polimérico MAGIC-f com a formulação modificada e os dados obtidos computacionalmente, a maior diferença encontrada foi de $12,5 \%$ para o corte realizado no centro da fonte, enquanto que para o corte em $0,5 \mathrm{~cm}$ na direção do topo do encapsulamento foi de $6,4 \%$.

\section{Conclusões}

Neste trabalho, foram realizadas comparações entre a reposta do gel polimérico MAGIC- $f$ com sua formulação modificada e os dados obtidos computacionalmente, através da simulação Monte Carlo com o código PENELOPE, para uma fonte clínica de Braquiterapia de alta taxa de dose.

Os resultados desse trabalho mostraram que, considerando-se 0 alto gradiente de dose dessas distribuições, o gel MAGIC- $f$ com formulação modificada é promissor para dosimetria em braquiterapia.

\section{Agradecimentos}

Os autores agradecem à CAPES pelo apoio financeiro; à Universidade de São Paulo; ao Hospital das Clínicas da Faculdade de Medicina de Ribeirão Preto - USP pela disposição dos aparelhos utilizados.

\section{Referências}

1. Sadeghi M, Saidi P, Tenreiro C. Applications of Monte Carlo methods in Biology, Medicine and other fiels of Science. India: InTech; 2011.

2. Papagiannis $P$, Pantelis $E$, Karaiskos $P$. Current state of the art brachytherapy treatment planning dosimetry algorithms. Brit J Radiol 2014; 87: 20140163.

3. Sempau J, Fernández-Varea JM, Acosta E, Salvat F. Experimental benchmarks of the Monte Carlo code PENELOPE. Nucl Instrum Meth B 2003; 207:107-123.

4. Romano S, Fusi F, Bolla E, Mencaglia AA, Mignani AG. Optical films for dosimetry in Radiotherapy. Proceedings of the Society of Photo-Optical Instrumentation Engineers (SPIE); 1996.

5. Peroni $\mathrm{Cm}$, Cirio R, Brusaco C, Dennis J, Givehchi $\mathrm{N}$ et al. A new 2-D ion chambre detector for Radiotherapy quality assurance. Proceedings of 49th annual meeting of the American-Society for therapeutic radiology and oncology; 2007; Los Angeles. Int J Rad Oncol Biol Phys 69(3): S733.

6. Haworth A, Butler DJ, Wifer L, Ebert MA, Todd SP et al. Comparison of TLD Calibration Methods for 1921r Dosimetry. J Appl Clin Med Phys 2013; 14(1): 4037. 
7. Atoobian $\mathrm{N}$, Asl AS, Poorbaygi $\mathrm{H}$, Javanshir MR. Gafchromic film dosimetry of a new HDR Ir-192 brachytherapy source. J Appl Med Phys 2016; 17(2): 194205.

8. Fazli Z, Sadeghi M, Zahmatkesh MH, Mahdavi SR, Tenreiro C. Dosimetric comparison between three dimensional treatment planning system, Monte Carlo simulation and gel dosimetry in Nasopharynx phantom for high dose rate Brachytherapy. J Cancer Res Ther 2013; 9(3): 402-9.

9. Teles P, Barros S, Cardoso S, Facure A, da Rosa LAR et al. A dosimetric study of prostate brachytherapy using Monte Carlo simulations with voxel phantom, measurements and a comparison with a treatment planning procedure. Radiat Prot Dosimetry 2015; 165(1-4): 482-7.

10. Wong CJ, Ackery T, He C, Patterson W, Powell CE et al. High-resolution measurements of small field beams using polymer gels. Appl Radiat Isto 2007; 65;1160-4.

11. De Deene $Y$, Walle R, Achten E, De Wagter C. Mathematical analysis and experimental investigation of noise in quantitative magnetic resonance imaging applied in polymer gel dosimetry. Signal Processing 1998; 70:85-101.

12. Carthwright LE, Suchowerska N, Yin Y, Lambert J, Haque M et al. Dose mapping of the rectal wall during Brahytherapy woth na array of scintillation dosimeters. Med Phys 2010; 37:2247-55.

13. Johnston H, Hllts $M$, Carrick J, Jirasek A. Na X-ray CT polymer gel dosimetry prototype: II. Gel characterization and clinical application. Phys Med Biol 2012; 57(10): 3155-75.

14. 14. Khoei S, Trapp JC, Langton CM. Quantitative evaluation of polymer gel dosimeters by croadhand ultrasound attenuation. J Phys Conf Ser 2013; 444:012084.

15. Goetsch SJ, Attix FH, Pearson DW, Thomadsen BR. Calibration of 1921r high-dose-rate afterloading systems. Med Phys 1991; 18(3): 462-7.

16. 16. Salvajoli JV, Souhami L, Faria SL. Radioterapia em Oncologia. São Paulo: Atheneu; 2013.

17. Zilio VO, Joneja OP, Popowski Y, Chawla R. Dosimetric characterization of radioactive sources employed in prostate câncer therapy. Brachytherapy 2004; 3:201-14.

18. Baltas D, Giannouli S, Garbi A, Diakonos F, Geramani K et al. Application of of the Monte Carlo Integration (MCl) method for calculation of the anisotropy of 192/r Brachytherapy sources. Phys Med Biol 1998; 43(6): 17831801.

19. Pavoni JF, Pije TL, Snow J, DeWerd LA, Baffa O. Dosimetria tridimensional usando gel MAGIC com Formaldeído. Rev Bras Fis Med 2010; 4(1): 15-8.

\section{Contato:}

Ana Luíza Quevedo

Universidade de São Paulo / Departamento de Física

Av. Bandeirantes, 3900

14040-901 Ribeirão Preto, SP, Brasil

anaquevedo@usp.br 